\title{
Quadratic stochastic operators on Banach lattices
}

\author{
Michał Badocha $^{1}$ - Wojciech Bartoszek ${ }^{1}$
}

Received: 9 March 2017 / Accepted: 1 September 2017 / Published online: 18 September 2017 C The Author(s) 2017. This article is an open access publication

\begin{abstract}
We study the convergence of iterates of quadratic stochastic operators that are mean monotonic. They are defined on the convex set of probability measures concentrated on a weakly compact order interval $S=[0, f]$ of a fixed Banach lattice $F$. We study their regularity and identify the limits of trajectories either as the "infimum" or "supremum" of the support of initial distributions.
\end{abstract}

Keywords Quadratic stochastic operator · Nonlinear Markov process · Weak convergence Phenotypic evolution

Mathematics Subject Classification Primary 60J35; Secondary 47H20 · 92D25

\section{Introduction}

The theory of quadratic stochastic operators (q.s.o.) is rooted in works of Bernstein (cf. [8,9]). Their importance was rediscovered in 1950 s by S. Ulam. His seminal report [28] prompted for many theoretical publications (cf. [4-7, 11,12, 16, 20,21,29]), monographs (cf. $[13,23]$ ) and computer supported mathematical projects (cf. $[3,14$, 15]) aiming to develop a unified theory illustrating (asymptotic) properties of q.s.o.. This subject has been intensively studied in mathematics and biology for almost three decades. In spite of that important questions have remained unsolved and the task is

$凶 \quad$ Wojciech Bartoszek

bartowk@mifgate.mif.pg.gda.pl

Michał Badocha

mbadocha@gmail.com

1 Department of Probability and Biomathematics, Gdańsk University of Technology, ul. Narutowicza 11/12, 80233 Gdańsk, Poland 
far from being completed. The asymptotic behavior of real or abstract mathematical models of biological systems, consisting with three or more biotypes, is not fully understood. We anticipate that q.s.o. will play in future an important role in genetics, population dynamics, social sciences as well as in other areas. Mathematically they perfectly suit to model the evolution of statistical configurations of all kinds of biotypes (genome, phenotypes e.t.c.) both for finite or continuous populations. The reader is referred to $[13,17]$ for a comprehensive and updated review of the topic. The list of very recent articles and online presentations is long. Let us only mention $[18,19$, $25,26]$, where the notion of quadratic stochastic operators is put into abstract vector (Banach) spaces with specific order or norm structures. In [24] the author studies nonhomogeneous Markov chains on ordered Banach spaces which are strongly linked to some picture of quadratic stochastic operators. Namely, as it has been proved in [7], generated by a quadratic stochastic operator $\mathbb{V}$ the so-called associated Markov chains may be efficiently used to determine the behavior of $\mathbb{V}^{n}$.

There has been much interest in recent years in self-organizing search methods in the q.s.o. field. Recently Ganikhodjaev, Saburov and Muhitdinov (see [15]) have generalized the notion of q.s.o. to bilinear forms on $\sigma$-additive measures on $[0,1]$. In particular, points of the unit interval $[0,1]$ serve to code (continuum valued) traits attributed to each individual from a considered population. As usual children inherit their traits from (two) parents, who mate randomly. The paper [15] drops the Mendelian paradigm and introduces trends (however, only in a one-dimensional direction), which are steady in time. The authors proved in [15] regularity in the case when there is no mutation and an inherited trait comes (randomly) from one of its parents. In this paper we propose further extensions. In particular, we obtain regularity under essentially weaker constraints. Moreover, our methods applied to theorems and examples from [15] simplify existing proofs.

Instead of one particular trait we propose to characterize an individual by infinite dimensional set of parameters, encoded by vectors from an order interval $S=[0, f]$ in a fixed real Banach lattice $(F,\|\cdot\|, \leq)$. Let us very briefly recall basic notions necessary to formulate our results (regarding the theory of Banach lattices and other facts from functional analysis the reader is referred to [2] or [27]). A Banach space $(F,\|\cdot\|)$ equipped with a partial order $\leq$ is a Banach lattice if the lattice operations (the infimum $x \wedge y$ and the supremum $x \vee y$ ) are well defined in $F$, satisfy axioms of Riesz spaces and are compatible with the norm topology (cf. [2], pp. 4, 5, 181 or [27], pp. 47-52). In particular, $\|y\| \leq\|x\|$ whenever $|y| \leq|x|$ in $F$. The modulus in $F$ is defined as $|x|=x \vee(-x)$, and therefore $\||x|\|=\|x\|$ for all $x \in F$. The positive cone $F_{+}=\{x \in F: 0 \leq x\}$ is a (weakly) closed subset of $F$ (cf. [27], Proposition 5.2). Let us mention that for all $x, y \in F$ we have $x \vee y=\frac{x+y+|x-y|}{2}$, and $x \wedge y=\frac{x+y-|x-y|}{2}$. The order interval, with endpoints $a \leq b \in F$, is defined as $I_{a, b}=[a, b]=\{x \in F: a \leq x \leq b\}$. If $a=0$ and $f \in \bar{F}_{+}$, we simply write $I_{f}$ in place of $I_{0, f}=[0, f]$. In the case $F=\mathbb{R}$, with ordinary modulus $|\cdot|$, order intervals are classical segments $[a, b]=\{x \in \mathbb{R}: a \leq x \leq b\}$. It is well known that order intervals in Banach lattices are always bounded, convex and (weakly) closed. However, they are not weakly compact in general. Classical spaces $\left(L^{p}(\mu),\|\cdot\|_{p}\right)$ and $\left(C(K),\|\cdot\|_{\text {sup }}\right)$ are important examples of Banach lattices. It follows from the Banach- 
Alaoglu theorem that order intervals are weakly compact if $F$ is reflexive. In particular, if $F=L^{p}(\mu)$, where $1<p<\infty$ (using different arguments also in $L^{1}(\mu)$ ), then order intervals are weakly compact. However, this does not hold for $C(K)$ or $L^{\infty}(\mu)$. We recall that the weak topology on a Banach space $(F,\|\cdot\|)$ is the smallest topology $\mathcal{T}$ so that all norm continuous linear functionals $\xi \in F^{\prime}$ are $\mathcal{T}$ continuous. The weak topology is denoted by $\mathcal{T}_{w}$ and it is a locally convex topology generated by the basis consisting of neighborhoods $\mathcal{U}_{\xi_{1}, \ldots, \xi_{n}, \varepsilon}=\left\{u \in F:\left|\xi_{j}(u)\right|<\varepsilon, j=1, \ldots, n\right\}$ of the zero vector $0 \in F$, where $n \in \mathbb{N}, \xi_{1}, \ldots, \xi_{n} \in F^{\prime}$, and $\varepsilon>0$. The net $x_{\alpha}$ converges weakly to $x$ in $F$ if and only if for every fixed $\xi \in F^{\prime}$ we have $\lim _{\alpha} \xi\left(x_{\alpha}\right)=\xi(x)$. Weak compactness plays in the category of Banach lattices an important role (cf. [2], chapter 4.2).

In this paper we shall deal with order intervals (phase spaces) $S \subset F$, which are assumed to be compact and metrizable for the weak topology (in particular, by the Mazur theorem they are norm separable). Hence the weak and norm Borel structures on $S$ coincide. We denote the Borel $\sigma$ field in $S$ by $\mathcal{B}$. As usual $\delta_{x}$ stands for the Dirac measure at $x \in S$. Compactness of $S$ implies (cf. $\S 6$ in [10]) that the convex set $\mathcal{P}(S)$ of all probability measures $\mu$, on the measurable space $(S, \mathcal{B})$, is compact (metrizable) for the weak measure convergence. We recall that a sequence of probability measures $\mu_{n} \in \mathcal{P}(S)$ converges to $\mu \in \mathcal{P}(S)$ in the weak convergence of measures if $\lim _{n \rightarrow \infty} \int_{S} f d \mu_{n} \rightarrow \int_{S} f d \mu$ holds for all $\mathcal{T}_{w}$ continuous (bounded) functions $f: S \rightarrow \mathbb{R}$. Then we write $\mu_{n} \Rightarrow \mu$. In order not to overexploit the term "weak" (especially in two different contexts) we shall use the notion of the Fortet-Mourier norm. We recall that given a finite Borel (signed) measure $\mu$ on $S$, the Fortet-Mourier norm is defined as $\|\mu\|_{\mathrm{FM}}=\sup \left\{\left|\int f d \mu\right|: 0 \leq f \leq 1\right.$ and $\left.\operatorname{Lip} f \leq 1\right\}$ (cf. [22], p. 48). As the reader may guess, given a continuous function $f: S \rightarrow \mathbb{R}$

$$
\operatorname{Lip} f=\sup \left\{\frac{|f(x)-f(y)|}{\varrho_{S}(x, y)}: x \neq y \in S\right\},
$$

where $\varrho_{S}$ is a fixed metric on $S$ compatible with the relative weak topology $\left.\mathcal{T}_{w}\right|_{S}$ on the set $S$ (the choice of a specific metric $\varrho_{S}$ is not crucial). It is well known (see [22], p. 49 , theorem 1.46) that on the space $\mathcal{P}(S)$ the convergence $\mu_{n} \Rightarrow \mu$ holds if and only if $\left\|\mu_{n}-\mu\right\|_{\mathrm{FM}} \rightarrow 0$.

By $\operatorname{bar}(\mu)$ we denote the barycenter of $\mu$, as long as it exists. We understand it as the Pettis integral $\int_{F} z d \mu(z)$; i.e. $\xi(\operatorname{bar}(\mu))=\xi\left(\int_{F} z d \mu(z)\right)=\int_{F} \xi(z) d \mu(z)$ for all $\xi \in F^{\prime}$. Clearly $\operatorname{bar}(\mu)$ is well defined if the topological $\operatorname{support} \operatorname{supp}(\mu)$ is weakly compact (cf. [1], pp. 10-12).

We denote $i(\mu)=\inf \operatorname{supp}(\mu), s(\mu)=\sup \operatorname{supp}(\mu)$ and $I(\mu)=[i(\mu), s(\mu)]$ as long as all is well defined. For this, let us suppose that $F$ has an order continuous norm (cf. [2], pp. 185-186) and $\operatorname{supp}(\mu)$ is contained in an order interval $I_{a, b}=[a, b] \subseteq F_{+}$. Clearly, $r_{n}=x_{1} \wedge \cdots \wedge x_{n} \in I_{a, b}$, if $x_{j} \in \operatorname{supp}(\mu), n=1,2, \ldots$. Hence $\left\|r_{n}\right\| \geq\|a\|$ is bounded from below. Using induction method and the property of norm order continuity (cf. [2], Theorem 4.9, p. 186) we may construct a sequence $\left(x_{j}\right)_{j \geq 1}$ such that $\lim _{n \rightarrow \infty} r_{n}=r \in[a, b]$ exists and the norm $\|r\|$ is as small as possible (we can take a dense sequence $x_{j} \in \operatorname{supp}(\mu)$ actually). Notice that $r \leq x$ for all $x \in \operatorname{supp}(\mu)$. If $v \leq x$ for all $x \in \operatorname{supp}(\mu)$, then $v \leq x_{j}$ for all $j$, hence $v \leq r_{n}$ for all $n$ and finally 
$v \leq r$. In particular, $r=i(\mu)$ does exist (however not necessarily $r \in \operatorname{supp}(\mu)$ ). Similarly we obtain the existence of $s(\mu)$.

\section{Basics on quadratic stochastic operators}

Given a weakly compact metrizable set $S \subseteq F$, let $\mathbb{P}=\{P(x, y, \cdot)\}_{x, y \in S}$ be a family of set functions (defined on the measurable space $(S, \mathcal{B})$, where $\mathcal{B}$ stands for the Borel $\sigma$-field) satisfying:

(2.1) $P(x, y, \cdot)=P(y, x, \cdot) \in \mathcal{P}(S)$ for all $x, y \in S$,

(2.2) $S \times S \ni(x, y) \rightarrow P(x, y, A) \in[0,1]$ is measurable for every fixed $A \in \mathcal{B}$.

Then the system $\mathbb{P}=\{P(x, y, \cdot)\}_{x, y \in S}$ is called a quadratic transition probability function. If moreover it satisfies

(2.3) $S \times S \ni(x, y) \rightarrow P(x, y, \cdot) \in \mathcal{P}(S)$ is continuous, where $S \times S$ is endowed with the weak $\times$ weak topology and in $\mathcal{P}(S)$ we have the Fortet-Mourier norm $\|\cdot\|_{\mathrm{FM}}$,

then such a quadratic transition function is called Feller [clearly the condition (2.3) implies (2.2)].

Let us mention briefly, that quadratic transition functions can be used to define perhaps the simplest class of nonlinear Markov processes $\left\{\xi_{k}\right\}_{k \geq 0}$. We will not dwell on this subject but rather focus only on the evolution and weak limits of distributions $P\left(\xi_{k} \in \cdot\right)$. However, the problem of behavior of trajectories $\xi_{k}(\omega)$, or the rate of convergence, remains untouched.

Any measurable quadratic transition probability function $\mathbb{P}$ may be uplifted to a bilinear mapping of $\mathcal{P}(S)$. Namely, define

$$
\mathcal{P}(S) \times \mathcal{P}(S) \ni(\mu, v) \rightarrow Q(\mu, v)(\cdot)=\int_{S} \int_{S} P(x, y, \cdot) d \mu(x) d v(y) \in \mathcal{P}(S) .
$$

Definition 2.1 A quadratic stochastic operator (generated by the family $\mathbb{P}$ ) is defined as $\mathbb{V}(\mu)=Q(\mu, \mu)$. It is called Feller if the quadratic transition probability function $\mathbb{P}$ is Feller [i.e. when (2.3) holds].

It is not hard to prove (cf. [4,7]) that $\|\mathbb{V}(\mu)-\mathbb{V}(v)\|_{\mathrm{TV}} \leq 2\|\mu-v\|_{\mathrm{TV}}$ for all $\mu, v \in \mathcal{P}(S)$, so $\mathbb{V}$ is a continuous transformation for the total variation norm $\|\cdot\|_{\mathrm{TV}}$ topology. Moreover, if it is Feller, then $\mathbb{V}$ is continuous on $\mathcal{P}(S)$ for the Fortet-Mourier norm; i.e.

$$
\lim _{n \rightarrow \infty} \mathbb{V}\left(\mu_{n}\right)=\mathbb{V}\left(\lim _{n \rightarrow \infty} \mu_{n}\right) \text { in the norm }\|\cdot\|_{\mathrm{FM}}
$$

as long as $\mu_{n} \in \mathcal{P}(S)$ converges in the norm $\|\cdot\|_{\mathrm{FM}}$ Indeed, let $\left\|\mu_{n}-\mu\right\|_{\mathrm{FM}} \rightarrow 0$ and $g: S \rightarrow \mathbb{R}$ be weakly continuous. Then

$$
\int_{S} g(z) \mathbb{V}\left(\mu_{n}\right)(d z)=\int_{S} \int_{S} \int_{S} g(z) P(x, y, d z) d \mu_{n}(x) d \mu_{n}(y) .
$$


By (2.3) the function $S \times S \ni(x, y) \rightarrow \int_{S} g(z) P(x, y, d z)$ is weakly continuous. It is well known that convergence in the Fortet-Mourier norm is preserved if we bring it to direct products. In other words if $\left\|\mu_{n}-\mu\right\|_{\mathrm{FM}} \rightarrow 0$ and $\left\|v_{n}-v\right\|_{\mathrm{FM}} \rightarrow 0$ then $\left\|\mu_{n} \otimes v_{n}-\mu \otimes v\right\|_{\mathrm{FM}} \rightarrow 0$. Thus

$$
\begin{aligned}
& \lim _{n \rightarrow \infty} \int_{S} \int_{S} \int_{S} g(z) P(x, y, d z) d \mu_{n}(x) d \mu_{n}(y)=\int_{S} \int_{S} \int_{S} g(z) P(x, y, d z) d \mu(x) d \mu(y) \\
& =\int_{S} g(z) \int_{S} \int_{S} P(x, y, d z) d \mu(x) d \mu(y)=\int_{S} g(z) \mathbb{V}(\mu)(d z) .
\end{aligned}
$$

The natural notion of regularity, in the context of q.s.o., was introduced very early (see $[17,23])$. We adopt it for the Fortet-Mourier topology.

Definition 2.2 We say that a q.s.o. $\mathbb{V}: \mathcal{P}(S) \times \mathcal{P}(S) \rightarrow \mathcal{P}(S)$ is weakly regular if

$$
\lim _{n \rightarrow \infty}\left\|\mathbb{V}^{n}(\mu)-L(\mu)\right\|_{\mathrm{FM}}=0
$$

for every $\mu \in \mathcal{P}(S)$, where the limit measure $L(\mu) \in \mathcal{P}(S)$. If there exists a unique measure $\mu_{*} \in \mathcal{P}(S)$ such that

$$
\lim _{n \rightarrow \infty}\left\|\mathbb{V}^{n}(\mu)-\mu_{*}\right\|_{\mathrm{FM}}=0
$$

for all $\mu \in \mathcal{P}(S)$, then a q.s.o. $\mathbb{V}$ is called weak mixing.

Depending on the (topological) point of view different modes of convergence are distinguished. To connect our results with other contemporary studies we recall (cf. $[4,7])$ the following notions:

Definition 2.3 We say that a q.s.o. $\mathbb{V}: \mathcal{P}(S) \times \mathcal{P}(S) \rightarrow \mathcal{P}(S)$ is strongly regular if

$$
\lim _{n \rightarrow \infty}\left\|\mathbb{V}^{n}(\mu)-L(\mu)\right\|_{\mathrm{TV}}=0
$$

for every $\mu \in \mathcal{P}(S)$, where the limit measure $L(\mu) \in \mathcal{P}(S)$. If there exists a unique measure $\mu_{*} \in \mathcal{P}(S)$ such that

$$
\lim _{n \rightarrow \infty}\left\|\mathbb{V}^{n}(\mu)-\mu_{*}\right\|_{\mathrm{TV}}=0
$$

for all $\mu \in \mathcal{P}(S)$, then a q.s.o. $\mathbb{V}$ is called strong mixing.

The uniform convergence give us:

Definition 2.4 We say that a q.s.o. $\mathbb{V}: \mathcal{P}(S) \times \mathcal{P}(S) \rightarrow \mathcal{P}(S)$ is uniformly regular if 


$$
\lim _{n \rightarrow \infty} \sup _{\mu \in \mathcal{P}(S)}\left\|\mathbb{V}^{n}(\mu)-L(\mu)\right\|_{\mathrm{TV}}=0
$$

If there exists a unique measure $\mu_{*} \in \mathcal{P}(S)$ such that

$$
\lim _{n \rightarrow \infty} \sup _{\mu \in \mathcal{P}(S)}\left\|\mathbb{V}^{n}(\mu)-\mu_{*}\right\|_{\mathrm{TV}}=0
$$

then a q.s.o. $\mathbb{V}$ is called norm mixing.

Strong and norm mixing q.s.o. and their geometric structure have been recently described in [4,5,7]. The weak mixing is studied in [6]. However, Bartoszek et al. [6] is restricted to kernel quadratic stochastic operators. In particular, $P(x, y, \cdot)$ are assumed to be absolutely continuous with respect to the Lebesgue measure and additionally $\int z P(x, y, d z)=\frac{x+y}{2}$ for all $x, y$ (roughly speaking-the offspring is on average the mean of the parents). Such operators are called centred kernel q.s.o. On the other hand, the models considered in [6] generally apply to unbounded phase spaces. Their weak limit of $\mathbb{V}^{n}(f)$ may be (depending on $f$ ) both discrete (Dirac $\delta$ ) or other probability measures, depending to what extent the CLT works. Mathematical methods used in their proofs come from the theory of characteristic functions, making it difficult to obtain closed form statements about the limits. In our paper we focus solely on weak regularity, assuming bounded domains and we look what happens if the evolution has a trend. In particular, our quadratic transition probabilities are not centred. We may consider our approach as generalizations of [15] and complementary to [6].

If for all $x, y \in S$ the measure $P(x, y, \cdot)$ is a convex combination of $\delta_{x}, \delta_{y}$, then the q.s.o. $\mathbb{V}$ is called Volterra. Clearly the Volterra q.s.o. can only model the mutation free evolution. The Mendelian situation occurs when $P(x, y, \cdot)=0.5 \delta_{x}+0.5 \delta_{y}$. We notice that in the last case $\mathbb{V}(\mu)=\mu$ for all $\mu \in \mathcal{P}(S)$.

Following [15], when $S=[0,1]$ and $F=\mathbb{R}$, Volterra q.s.o. are called Lebesgue q.s.o. It has been proved (see Theorem 4.6 in [15]) that any Lebesgue q.s.o. such that $P(x, y, \cdot)=p \delta_{x \wedge y}(\cdot)+(1-p) \delta_{x \vee y}(\cdot)$, with $P(x, x, \cdot)=\delta_{x}(\cdot)$ is weakly regular, and the iterates $\mathbb{V}^{n}(\mu)$ converge to $\delta_{i(\mu)}$, if $0.5<p \leq 1$ or respectively to $\delta_{s(\mu)}$ if $0 \leq p<0.5$ (when $p=0.5$ then $\mathbb{V}$ is the identity transformation).

Our approach brings further extensions, with two folded generalizations. Firstly, in the case of Lebesgue operators $\mathbb{V}$ on $S=[0,1]$, we only assume that $P(x, y, \cdot)=$ $\alpha(x, y) \delta_{x \wedge y}(\cdot)+(1-\alpha(x, y)) \delta_{x \vee y}(\cdot)$, where $\alpha(x, y)>0.5$ for all $x \neq y \in[0,1]$ (or $\alpha(x, y)<0.5$ for all $x \neq y \in[0,1]$ respectively). We will show that this condition may be relaxed further (see our Corollaries 5.1 and 5.2). In particular, we do not require the Volterra condition. Secondly, we shall extend Ganikhodjaev's, Saburov's and Muhitdinov's results from [15] to general phase spaces, assuming that $S=I$ is a weakly compact order interval. Let us recall (cf. [2], Theorem 4.9) that order intervals in Banach lattices with order continuous norm are weakly compact. And finally, in general Banach lattices the notion of Lebesgue q.s.o. may split in different directions. For this let us only mention that $\alpha(x, y) \delta_{x \wedge y}(\cdot)+(1-\alpha(x, y)) \delta_{x \vee y}(\cdot)$ is not necessarily a convex combination of $\delta_{x}, \delta_{y}$. 


\section{Monotonicity of means and regularity}

Theorem 3.1 Let $I=[0, f] \subset F$ be a weakly metrizable order interval in a Banach lattice $(F,\|\cdot\|, \leq)$ with order continuous norm and let $\{P(x, y, \cdot)\}_{x, y \in I}$ be a quadratic transition probability function satisfying:

$$
\int_{I} z P(x, y, d z) \leq \frac{x+y}{2}, \text { for all } x, y \in I
$$

Then for every $\mu \in \mathcal{P}(I)$ the barycenters of $\mathbb{V}^{n}(\mu)$ converge in the norm to $c(\mu) \in I$. In particular $\lim _{n \rightarrow \infty} \int_{I} \xi(z) \mathbb{V}^{n}(\mu)(d z)=\xi(c(\mu))$ for every $\xi \in F^{\prime}$.

If additionally the transition probabilities $P(x, y, \cdot)$ are Feller and have the property that

(3.2) the equality in (3.1) implies $x=y$,

then $\lim _{n \rightarrow \infty} \mathbb{V}^{n}(\mu)=\delta_{c(\mu)}$ in the $\|\cdot\|_{\mathrm{FM}}$ norm.

If moreover,

(3.3) $\operatorname{supp} P(x, y, \cdot) \subseteq[x \wedge y, x \vee y]$ for all $x, y \in I$.

then $c(\mu) \in I(\mu)$.

Proof It is sufficient to evaluate the $\operatorname{limits}_{\lim } \rightarrow \infty \int_{I} \xi(z) V^{n}(\mu)(d z)$ only for positive $\xi \in F_{+}^{\prime}$, as the dual space $F^{\prime}=F_{+}^{\prime}-F_{-}^{\prime}$ is a Banach lattice too (cf. [2], Proposition 5.5). For every natural $n$

$$
\begin{aligned}
\xi & \left(\operatorname{bar}\left(\mathbb{V}^{n+1}(\mu)\right)\right)=\int_{[0, f]} \xi(z) \mathbb{V}^{n+1}(\mu)(d z) \\
= & \int_{[0, f]} \xi(z) \int_{[0, f]} \int_{[0, f]} P(x, y, d z) \mathbb{V}^{n}(\mu)(d x) \mathbb{V}^{n}(\mu)(d y) \\
= & \int_{[0, f]} \int_{[0, f]}\left(\xi\left(\int_{[0, f]} z P(x, y, d z)\right)\right) \mathbb{V}^{n}(\mu)(d x) \mathbb{V}^{n}(\mu)(d y) \\
\leq & \int_{[0, f]} \int_{[0, f]} \xi\left(\frac{x+y}{2}\right) \mathbb{V}^{n}(\mu)(d x) \mathbb{V}^{n}(\mu)(d y) \\
= & \int_{[0, f]} \int_{[0, f]} \xi\left(\frac{x}{2}\right) \mathbb{V}^{n}(\mu)(d x) \mathbb{V}^{n}(\mu)(d y) \\
& +\int_{[0, f]} \int_{[0, f]} \xi\left(\frac{y}{2}\right) \mathbb{V}^{n}(\mu)(d x) \mathbb{V}^{n}(\mu)(d y) \\
= & \int_{[0, f]} \xi(z) \mathbb{V}^{n}(\mu)(d z)=\xi\left(\operatorname{bar}\left(\mathbb{V}^{n}(\mu)\right)\right) .
\end{aligned}
$$

Hence (cf. [2], Proposition 5.5), $0 \leq \operatorname{bar}\left(\mathbb{V}^{n+1}(\mu)\right) \leq \operatorname{bar}\left(\mathbb{V}^{n}(\mu)\right)$ for all $n \in \mathbb{N}$, as the order in $F^{\prime}$ is induced by $F_{+}^{\prime}=\left\{\xi \in F^{\prime}: \xi(x) \geq 0\right.$ for all positive $\left.x \in F_{+}\right\}$. The barycenters of $\mathbb{V}^{n}(\mu)$ form a nonincreasing sequence in $I$. It follows (cf. [2], Theorem 4.9 , p. 186) that $\operatorname{bar}\left(\mathbb{V}^{n}(\mu)\right)$ converges (in the norm) to a vector $c(\mu) \in[0, f]$. 
Let $n_{j} \nearrow \infty$ be any increasing sequence such that $\mathbb{V}^{n}(\mu)$ converges weakly to some $v \in \mathcal{P}(S)$. By the Feller assumption

$$
\mathbb{V}(v)=\mathbb{V}\left(\lim _{j \rightarrow \infty} \mathbb{V}^{n_{j}}(\mu)\right)=\lim _{j \rightarrow \infty} \mathbb{V}^{n_{j}+1}(\mu)
$$

It follows that

$$
c(\mu)=\lim _{j} \operatorname{bar}\left(\mathbb{V}^{n_{j}+1}(\mu)\right)=\operatorname{bar}(\mathbb{V}(v)) \leq \operatorname{bar}(v)=\lim _{j} \operatorname{bar}\left(\mathbb{V}^{n_{j}}(\mu)\right)=c(\mu) .
$$

We obtain $\operatorname{bar}(\mathbb{V}(v))=\operatorname{bar}(v)$. Hence for any positive $\xi \in F^{\prime}$ we get

$$
\begin{aligned}
0 & =\int_{I} \xi(x) v(d x)-\int_{I} \xi(x) \mathbb{V}(v)(d x) \\
& =\int_{I} \int_{I} \xi\left(\frac{x+y}{2}\right) v(d x) v(d y)-\int_{I} \int_{I} \int_{I} \xi(z) P(x, y, d z) v(d x) v(d y) \\
& =\int_{I} \int_{I} \xi\left(\frac{x+y}{2}-\int_{I} z P(x, y, d z)\right) v(d x) v(d y) .
\end{aligned}
$$

By positivity of $\xi$ and condition (3.1) we get $\xi\left(\frac{x+y}{2}-\int_{I} z P(x, y, d z)\right)=0$ for $v \times v$ almost all $(x, y)$. Now, applying the additional assumption (3.2), we get $x=y$ for $v \times v$ almost all $(x, y)$. Hence $v=\delta_{c(\mu)}$. Since $n_{j}$ was arbitrary (with only restriction that $\mathbb{V}^{n_{j}}(\mu)$ converges), thus $\lim _{n \rightarrow \infty} \mathbb{V}^{n}(\mu)=\delta_{c(\mu)}$.

Finally applying (3.3) we notice that $\operatorname{supp} \mathbb{V}(\mu) \subseteq I(\mu)$. Iterating this inclusion we obtain

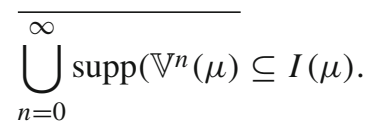

We obtain $c(\mu) \in I(\mu)$.

The next result concerns a specific class of quadratic transition probabilities. They generalize the notion of Lebesgue quadratic stochastic operators introduced in [15].

Definition 3.2 Let $S=[0, f] \subset F$ be an order interval in a Banach lattice $F$. A quadratic transition probability family $\{P(x, y, \cdot)\}_{x, y \in S}$ is called order cramped if

$$
P(x, y, \cdot)=\alpha(x, y) \delta_{x \wedge y}+\beta(x, y) \delta_{x \vee y}+\frac{1-\alpha(x, y)-\beta(x, y)}{2} \delta_{x}+\frac{1-\alpha(x, y)-\beta(x, y)}{2} \delta_{y},
$$

where Borel functions $\alpha, \beta:[0, f] \times[0, f] \rightarrow[0,1]$ are symmetric (i.e. $\alpha(x, y)=$ $\alpha(y, x)$ and $\beta(x, y)=\beta(y, x))$, and satisfy $\alpha(x, y)+\beta(x, y) \leq 1$. The corresponding quadratic stochastic operator $\mathbb{V}$ also is called order cramped. If $\alpha(x, y)+\beta(x, y)=1$ then (following [15]) we call such operators Lebesgue. 
Obviously $\operatorname{supp}(P(x, y, \cdot)) \subseteq[x \wedge y, x \vee y]$. If $F=\mathbb{R}$ then we obtain a Volterra q.s.o.. Finally if $\alpha, \beta$ are weakly continuous on $S \times S$, then we get a Feller order cramped q.s.o..

Theorem 3.3 Let $\mathbb{V}$ be an order cramped quadratic stochastic operator defined on an order interval $I=[0, f]$ of a real Banach lattice $F$ with order continuous norm. If I is weakly metrizable, the functions $\alpha$ and $\beta$ are weakly continuous and $\alpha(x, y)>\beta(x, y)$ if $x \neq y$, then the corresponding q.s.o. $\mathbb{V}$ is weakly regular, and for all $\mu \in \mathcal{P}(\mu)$

$$
\lim _{n \rightarrow \infty}\left\|\mathbb{V}^{n}(\mu)-\delta_{c(\mu)}\right\|_{\mathrm{FM}}=0
$$

where $c(\mu) \in I(\mu)$.

Proof It suffices to verify the condition (3.1) of Theorem 3.1. For this

$$
\begin{aligned}
\int_{I} z P(x, y, d z)= & \alpha(x, y)(x \wedge y)+\beta(x, y)(x \vee y)+\frac{1-\alpha(x, y)-\beta(x, y)}{2}(x+y) \\
= & \alpha(x, y) \frac{x+y-|x-y|}{2}+\beta(x, y) \frac{x+y+|x-y|}{2} \\
& -(\alpha(x, y)+\beta(x, y)) \frac{x+y}{2}+\frac{x+y}{2} \\
= & \frac{x+y}{2}-\frac{\alpha(x, y)-\beta(x, y)}{2}|x-y| \leq \frac{x+y}{2}
\end{aligned}
$$

with equality only if $x=y$. By Theorem 3.1, for all $\mu \in \mathcal{P}(I)$ we obtain the convergence $\lim _{n \rightarrow \infty} \mathbb{V}^{n}(\mu)=\delta_{c(\mu)}$ in the $\|\cdot\|_{\mathrm{FM}}$ norm. We notice that, if $x, y \in \operatorname{supp}(\mu)$, then $\operatorname{supp}(P(x, y, \cdot)) \subseteq I(\mu)$. Hence $\operatorname{supp}(\mathbb{V}(\mu)) \subseteq I(\mu)$ and by induction $\operatorname{supp}\left(\mathbb{V}^{n}(\mu)\right) \subseteq I(\mu)$ for all $n \in \mathbb{N}$. It follows that $c(\mu) \in I(\mu)$.

\section{Finite dimensional case}

In the finite dimensional case, when $F=\mathbb{R}^{d}$ and the q.s.o. is Lebesgue, the last result may be strengthen. We assume that in $\mathbb{R}^{d}$ we have the standard order, so the positive cone is $\mathbb{R}_{+}^{d}=\left\{\left(x_{1}, \ldots, x_{d}\right): x_{j} \geq 0, j=1, \ldots, d\right\}$. The lattice norm $\|\cdot\|$ may be taken arbitrary as all norms on finite dimensional vector spaces are equivalent (or apply Corollary 4.4 from [2]).

Theorem 4.1 Let $I=[0, f]$ be a an order interval in the Banach lattice $F=\mathbb{R}^{d}$ and $P(x, y, \cdot)=\alpha(x, y) \delta_{x \wedge y}+\beta(x, y) \delta_{x \vee y}$, where $\alpha(x, y)=\alpha(y, x), \beta(x, y)=$ $\beta(y, x)$ are continuous functions such that $\alpha(x, y)>\beta(x, y)$ if $x \neq y$ and satisfy $\alpha(x, y)+\beta(x, y)=1$. Then the corresponding Lebesgue q.s.o. generated by $\mathbb{P}$ is weakly regular and for every $\mu \in \mathcal{P}(I)$

$$
\lim _{n \rightarrow \infty}\left\|\mathbb{V}^{n}(\mu)-\delta_{i(\mu)}\right\|_{\mathrm{FM}}=0
$$


Proof By Theorem 3.3 the sequence $\mathbb{V}^{n}(\mu)$ converges in $\|\cdot\|_{F M}$ to $\delta_{c(\mu)}$, where $c(\mu) \in[i(\mu), s(\mu)]$. Define $W_{u}=[i(\mu), u]$, where $i(\mu)<u \leq s(\mu)$ and set $D_{u}=$ $I \backslash W_{u}$. Then

$$
\begin{aligned}
\mathbb{V}(\mu)\left(W_{u}\right)= & \int_{W_{u}} \int_{W_{u}} P\left(x, y, W_{u}\right) d \mu(x) d \mu(y)+2 \int_{W_{u}} \int_{D_{u}} P\left(x, y, W_{u}\right) d \mu(x) d \mu(y) \\
& +\int_{D_{u}} \int_{D_{u}} P\left(x, y, W_{u}\right) d \mu(x) d \mu(y) \\
\geq & \mu\left(W_{u}\right)^{2}+2 \int_{W_{u}} \int_{D_{u}} \alpha(x, y) d \mu(x) d \mu(y) \\
\geq & \mu\left(W_{u}\right)^{2}+\mu\left(W_{u}\right) \mu\left(D_{u}\right)=\mu\left(W_{u}\right) .
\end{aligned}
$$

Clearly $i(\mathbb{V}(\mu))=i(\mu)$. Therefore, we can iterate the above estimation to obtain

$$
\mu\left(W_{u}\right) \leq \mathbb{V}(\mu)\left(W_{u}\right) \leq \cdots \leq \mathbb{V}^{n}(\mu)\left(W_{u}\right) \leq \cdots
$$

We have already noticed that $i(\mu)=\lim _{n \rightarrow \infty} r_{n}$, where $r_{n}=x_{1} \wedge \cdots \wedge x_{n}$, for some sequence $x_{j} \in \operatorname{supp}(\mu)$. It follows from the definition of Lebesgue quadratic transition function that $r_{n} \in \operatorname{supp}\left(\mathbb{V}^{n}(\mu)\right)$. Now for arbitrarily fixed $\varepsilon>0$ we find $k \geq 0$ such that $\left\|i(\mu)-r_{k}\right\|<\varepsilon$ and define $u_{k}=(1-\varepsilon) r_{k}+\varepsilon s(\mu)$. We shall show that $\mathbb{V}^{k}(\mu)\left(W_{u_{k}}\right)>0$. Let $J=\left\{j: r_{k, j}=s(\mu)_{j}\right\} \subseteq\{1, \ldots, d\}$. Then for every $j \in J$ we have $u_{k, j}=s(\mu)_{j}$ as well. Define $\varrho_{*}=\min \left\{s(\mu)_{j}-r_{k, j}: j \notin J\right\}$. Clearly the set $\mathcal{U}_{\varrho}\left(r_{k}\right)=\left\{x \in I:\left\|x-r_{k}\right\|_{\infty}<\varrho\right\}$ is an open neighborhood of $r_{k}$ (both for $\|\cdot\|$ and $\|\cdot\|_{\infty}$ norms) in the relative topology on $I$. Notice that, whenever $\varrho<\varepsilon \varrho_{*}$, then $\mathcal{U}_{\varrho}\left(r_{k}\right) \subseteq\left[i(\mu), u_{k}\right]=W_{u_{k}}$. In fact, for all $j \in J$ if $x \in \mathcal{U}_{\varrho}\left(r_{k}\right)$, then $x_{j} \leq s(\mu)_{j}=u_{k, j}$. On the other hand, if $j \notin J$, then

$$
\begin{aligned}
& x_{j}<r_{k, j}+\varrho=u_{k, j}+\varepsilon r_{k, j}-\varepsilon s(\mu)_{j}+\varrho=u_{k, j}-\varepsilon\left(s(\mu)_{j}-r_{k, j}\right)+\varrho \\
& \quad \leq u_{k, j}-\varepsilon \varrho_{*}+\varrho<u_{k, j} .
\end{aligned}
$$

Hence $0<\mathbb{V}^{k}(\mu)\left(\mathcal{U}_{\varrho}\left(r_{k}\right)\right) \leq \mathbb{V}^{k}(\mu)\left(W_{u_{k}}\right)$. By monotonicity we obtain

$$
\lim _{n \rightarrow \infty} \mathbb{V}^{n}(\mu)\left(\left[i(\mu), u_{k}\right]\right) \geq \mathbb{V}^{k}(\mu)\left(W_{u_{k}}\right)>0 .
$$

This implies $c(\mu) \in\left[i(\mu), u_{k}\right]$ and by the triangle inequality

$$
\begin{gathered}
\|c(\mu)-i(\mu)\| \leq\left\|u_{k}-i(\mu)\right\| \leq\left\|u_{k}-r_{k}\right\| \\
+\left\|r_{k}-i(\mu)\right\|<\varepsilon\|f\|+\varepsilon=\varepsilon(1+\|f\|) .
\end{gathered}
$$

We get $c(\mu)=i(\mu)$ as $\varepsilon>0$ may be as small as we wish.

In the finite dimensional case the Lebesgue quadratic operators may be generalized further. If $J \subseteq\{1,2, \ldots, d\}$ we introduce 2 -argument operations $\wedge_{J 1}, \vee_{J 1}, \wedge_{J 2}$ and $\vee_{J 2}$. Namely, if $x, y \in \mathbb{R}^{d}$ we define 


$$
\begin{aligned}
& (x \wedge J 1 y)_{k}= \begin{cases}(x \wedge y)_{k} & \text { if } k \in J \\
x_{k} & \text { if } k \notin J .\end{cases} \\
& \left(x \vee_{J 1} y\right)_{k}= \begin{cases}(x \vee y)_{k} & \text { if } k \in J \\
x_{k} & \text { if } k \notin J .\end{cases} \\
& (x \wedge J 2 y)_{k}= \begin{cases}(x \wedge y)_{k} & \text { if } k \in J \\
y_{k} & \text { if } k \notin J .\end{cases} \\
& \left(x \vee_{J 2} y\right)_{k}= \begin{cases}(x \vee y)_{k} & \text { if } k \in J \\
y_{k} & \text { if } k \notin J .\end{cases}
\end{aligned}
$$

We notice that if $J=\emptyset$ then $x \wedge_{\emptyset 1} y=x=x \vee_{\emptyset 1} y, x \wedge_{\emptyset 2} y=y=x \vee_{\emptyset 2} y$. On the other hand, if $J=\{1,2, \ldots d\}$ then $x \wedge_{\{1,2, \ldots, d\} 1} y=x \wedge y=x \wedge_{\{1,2, \ldots, d\} 2} y$ and $x \vee\{1,2, \ldots, d\} 1 y=x \vee y=x \vee\{1,2, \ldots, d\} 2 y$.

Let $\alpha_{J}(x, y)=\alpha_{J}(y, x)$ and $\beta_{J}(x, y)=\beta_{J}(y, x)$ be continuous functions on the order interval $[0, f]^{2} \subseteq \mathbb{R}^{d} \times \mathbb{R}^{d}$, where the index $J$ runs over all subsets of $\{1,2, \ldots, d\}$. If $\sum_{J}\left(\alpha_{J}(x, y)+\beta_{J}(x, y)\right)=\frac{1}{2}$, then

$$
\begin{aligned}
P(x, y, \cdot)= & \sum_{J}\left(\alpha_{J}(x, y) \delta_{x \wedge J 1} y+\alpha_{J}(x, y) \delta_{x \wedge J 2} y\right. \\
& \left.+\beta_{J}(x, y) \delta_{x \vee_{J 1} y}+\beta_{J}(x, y) \delta_{x \vee_{J 2} y}\right)
\end{aligned}
$$

defines properly a quadratic probability transition function. From the biological point of view these operators may model mutations in configurations described by coordinate subsets $J$.

Proposition 4.2 Let $\mathbb{P}$ be a quadratic probability transition function defined above acting on the finite dimensional interval $I \subset \mathbb{R}^{d}$, where $\mathbb{R}^{d}$ is a Banach lattice with ordinary coordinate order. If for all $J \subseteq\{1,2, \ldots, d\}$ we have $\alpha_{J}(x, y)>\beta_{J}(x, y)$ if $x \neq y$, then for every $\mu \in \mathcal{P}(I)$ we have

$$
\lim _{n \rightarrow \infty}\left\|\mathbb{V}^{n}(\mu)-\delta_{c(\mu)}\right\|_{\mathrm{FM}}=0
$$

where $c(\mu) \in[i(\mu), s(\mu)]$.

Proof We will verify conditions (3.1), (3.2) and (3.3) from Theorem 3.1. (3.3) follows directly from the definition of $P(x, y, \cdot)$. Given a vector $x \in I$ and $J \subseteq\{1,2, \ldots, d\}$, let us define a vector $\left.x\right|_{J} \in \mathbb{R}^{d}$ by $\left(\left.x\right|_{J}\right)_{k}=x_{k}$ if $k \in J$, and $\left(\left.x\right|_{J}\right)_{k}=0$ if $k \in J^{c}=$ $\{1,2, \ldots, d\} \backslash J$. We have

$$
\begin{aligned}
& \int_{I} z P(x, y, d z) \\
& \quad=\sum_{J}\left(\alpha_{j}(x, y)\left(x \wedge_{J 1} y+x \wedge_{J 2} y\right)+\beta_{J}(x, y)\left(x \vee_{J 1} y+x \vee_{J 2} y\right)\right)
\end{aligned}
$$




$$
\begin{aligned}
= & \sum_{J}\left(\left.\alpha_{J}(x, y)(x \wedge y)\right|_{J}+\left.\alpha_{J}(x, y) x\right|_{J^{c}}+\left.\alpha_{J}(x, y)(x \wedge y)\right|_{J}+\left.\alpha_{J}(x, y) y\right|_{J^{c}}\right. \\
& \left.+\left.\beta_{J}(x, y)(x \vee y)\right|_{J}+\left.\beta_{J}(x, y) x\right|_{J^{c}}+\left.\beta_{J}(x, y)(x \vee y)\right|_{J}+\left.\beta_{J}(x, y) y\right|_{J^{c}}\right) \\
= & \sum_{J} 2 \alpha_{J}(x, y)\left(\left.(x \wedge y)\right|_{J}-\left.\left(\frac{x+y}{2}\right)\right|_{J}\right) \\
& +2 \beta_{J}(x, y)\left(\left.(x \vee y)\right|_{J}-\left.\left(\frac{x+y}{2}\right)\right|_{J}\right)+\alpha_{J}(x, y)(x+y)+\beta_{J}(x, y)(x+y) \\
= & \sum_{J}\left(2\left(\alpha_{J}(x, y)-\beta_{J}(x, y)\right)\left((x \wedge y)_{J}-\left.\left(\frac{x+y}{2}\right)\right|_{J}\right)\right. \\
& \left.+2 \beta_{J}(x, y)\left(\left.(x \wedge y)\right|_{J}-\left.\left(\frac{x+y}{2}\right)\right|_{J}+\left.(x \vee y)\right|_{J}-\left.\left(\frac{x+y}{2}\right)\right|_{J}\right)\right) \\
& +\sum_{J}\left(\alpha_{J}(x, y)+\beta_{J}(x, y)\right)(x+y) \\
= & \sum_{J}\left(2\left(\alpha_{J}(x, y)-\beta_{J}(x, y)\right)\left((x \wedge y)_{J}-\left.\left(\frac{x+y}{2}\right)\right|_{J}\right)+0\right) \\
& +\frac{1}{2}(x+y) \leq \frac{x+y}{2},
\end{aligned}
$$

as $2\left(\alpha_{J}(x, y)-\beta_{J}(x, y)\right)\left((x \wedge y)_{J}-\left.\left(\frac{x+y}{2}\right)\right|_{J} \leq 0\right.$ for all $J$. Hence (3.1) is satisfied. If $x \neq y$ then $(x \wedge y)_{J}-\left.\left(\frac{x+y}{2}\right)\right|_{J} \supsetneqq 0$ for some $J \subseteq\{1,2, \ldots, d\}$. Hence (3.2) holds. Applying Theorem 3.1 we end the proof.

\section{Final conclusions and examples}

The following results are strictly related to [15]. Under extra assumptions we identify $\lim _{n \rightarrow \infty} \mathbb{V}^{n}(\mu)$ as $\delta_{i(\mu)}$ (or $\delta_{s(\mu)}$ respectively). We start with discussing classical Lebesgue q.s.o. introduced in [15]; i.e. consider $F=\mathbb{R}$ and $I=[0,1]$. The transition functions are defined as

$$
P(x, y, \cdot)=\alpha(x, y) \delta_{x \wedge y}+\beta(x, y) \delta_{x \vee y},
$$

where the functions $\alpha, \beta$ are symmetric on $I \times I$ and Borel measurable (continuity is not required), and $\alpha+\beta=1$. In [15] both $\alpha(x, y) \equiv \alpha=$ const and $\beta(x, y) \equiv \beta=$ const.

Theorem 5.1 Let $\mathbb{V}$ be the Lebesgue q.s.o. defined by $P(x, y, \cdot)$ as above, where $\alpha(x, y)>\beta(x, y)$ if $x \neq y$. If for some $\varepsilon>0$ and $0<\varrho<\frac{1}{2}$ the measure $\mu \in \mathcal{P}([0,1])$ satisfies

$$
\alpha(x, y)>\frac{1}{2}+\varrho \text { if } x \wedge y \in[i(\mu), i(\mu)+\varepsilon],
$$

then $\lim _{n \rightarrow \infty}\left\|\mathbb{V}^{n}(\mu)-\delta_{i(\mu)}\right\|_{\mathrm{FM}}=0$. 
Proof For a fixed $0<\tau<\varepsilon$ we set

$$
\gamma_{n}=\mathbb{V}^{n}(\mu)([i(\mu), i(\mu)+\tau]) .
$$

We get

$$
\begin{aligned}
\gamma_{n+1}= & \mathbb{V}^{n+1}(\mu)([i(\mu), i(\mu)+\tau]) \\
\geq & \left(\mathbb{V}^{n}(\mu)([i(\mu), i(\mu)+\tau])\right)^{2} \\
& +2\left(\frac{1}{2}+\varrho\right) \mathbb{V}^{n}(\mu)([i(\mu), i(\mu)+\tau]) \mathbb{V}^{n}(\mu)((i(\mu)+\tau, s(\mu)]) \\
\geq & \gamma_{n}^{2}+2\left(\frac{1}{2}+\varrho\right) \gamma_{n}\left(1-\gamma_{n}\right) .
\end{aligned}
$$

Clearly $\gamma_{0}=\mu([i(\mu), i(\mu)+\tau])>0$. Notice that $\varphi^{o n}(\mu([i(\mu), i(\mu)+\tau])) \leq \gamma_{n} \leq 1$, where the quadratic function $\varphi:[0,1] \rightarrow[0,1]$ is defined by the formula $\varphi(t)=$ $t^{2}+2\left(\frac{1}{2}+\varrho\right) t(1-t)$. Obviously the iterates $\lim _{n \rightarrow \infty} \varphi^{\circ n}(t)=1$ for all $0<t \leq 1$. Thus $\lim _{n \rightarrow \infty} \gamma_{n}=1$. It follows that $\lim _{n \rightarrow \infty} \mathbb{V}^{n}(\mu)([i(\mu), i(\mu)+\tau])=1$, where $\tau>0$ may be as small as we wish. We have proved $\lim _{n \rightarrow \infty} \mathbb{V}^{n}(\mu)=\delta_{i(\mu)}$.

By symmetry the following version of weak regularity holds;

Theorem 5.2 Let $\mathbb{V}$ be the Lebesgue q.s.o. defined by $P(x, y, \cdot)$ as above, where $\alpha(x, y)<\beta(x, y)$ if $x \neq y$. If for some $\varepsilon>0$ and $0<\varrho<\frac{1}{2}$ the measure $\mu \in \mathcal{P}([0,1])$ satisfies

$$
\beta(x, y)>\frac{1}{2}+\varrho \text { if } x \vee y \in[s(\mu)-\varepsilon, s(\mu)],
$$

then $\lim _{n \rightarrow \infty}\left\|\mathbb{V}^{n}(\mu)-\delta_{s(\mu)}\right\|_{\mathrm{FM}}=0$.

Even in the one-dimensional case, when $F=\mathbb{R}$ and $I=[0,1]$, the question on weak regularity of q.s.o. is nontrivial. In particular, it is a challenge to find a pattern of general limit measures. In the example below we see that the condition on the global mean monotonicity may be relaxed.

Example 5.3 Let $I=[0,1] \subset \mathbb{R}$. Given $0<u<v<1$ and Borel functions $p:[0, u) \rightarrow\left(\frac{1}{2}+\varrho, 1\right], q:(v, 1] \rightarrow\left[0, \frac{1}{2}-\varrho\right)$, where $0<\varrho<\frac{1}{4}$, let us define a symmetric function:

$$
\alpha(x, y)= \begin{cases}p(x), & \text { for } 0 \leq x<u, x \leq y \leq 1 \\ \frac{1}{2}, & \text { for } u \leq x \leq v, x \leq y \leq 1 \\ q(x), & \text { for } v<x \leq 1, x \leq y \leq 1\end{cases}
$$

Let $\mathbb{V}$ be the Lebesgue q.s.o. corresponding to $\alpha$ (i.e. $P(x, y, \cdot)=\alpha(x, y) \delta_{x \wedge y}+$ $\left.(1-\alpha(x, y)) \delta_{x \vee y}\right)$. We recall that intervals $[a, b] \subseteq[0,1]$ are invariant for Lebesgue q.s.o. $\mathbb{V}$ (i.e. if $\operatorname{supp}(\mu) \subseteq[a, b]$ then $\operatorname{supp}(\mathbb{V}(\mu)) \subseteq[a, b])$. Hence $\{P(x, y, \cdot)\}$ 
restricted to $[a, b]$ generates a q.s.o. which coincides with $\left.\mathbb{V}\right|_{\mathcal{P}([a, b])}$. The same trick which is used in the proof of Theorem 5.1 gives the convergence of $\gamma_{n}=$ $\mathbb{V}^{n}(\mu)([i(\mu), i(\mu)+\tau)) \rightarrow 1$, for every probability Borel measure $\mu$ on $[0,1]$ such that $\mu([0, u))>0$. It follows that $\lim _{n \rightarrow \infty}\left\|\mathbb{V}^{n}(\mu)-\delta_{i(\mu)}\right\|_{\mathrm{FM}}=0$. On the other hand if $\mu((v, 1])=1$, then by the Theorem 5.2 we obtain $\lim _{n \rightarrow \infty}\left\|\mathbb{V}^{n}(\mu)-\delta_{s(\mu)}\right\|_{\mathrm{FM}}=0$.

More generally, if $\mu([u, 1])=1$, then $\lim _{n \rightarrow \infty} \mathbb{V}^{n}(\mu)=\left.\mu\right|_{[u, v]}+\mu((v, 1]) \delta_{s(\mu)}$ in the $\|\cdot\|_{\mathrm{FM}}$ norm. Indeed, for every Borel $A \subseteq[u, v]$ we have

$$
\begin{aligned}
\mathbb{V}(\mu)(A)= & \int_{A} \int_{A} P(x, y, A) d \mu(x) d \mu(y)+2 \int_{A} \int_{A^{c}} P(x, y, A) d \mu(x) d \mu(y) \\
& +\int_{A^{c}} \int_{A^{c}} P(x, y, A) d \mu(x) d \mu(y) \\
= & \mu^{2}(A)+\mu(A) \mu\left(A^{c}\right)=\mu(A) .
\end{aligned}
$$

By induction, for every natural $n$ and every Borel $A \subseteq[u, v]$, we have $\mathbb{V}^{n}(\mu)(A)=$ $\mu(A)$. Hence the sequence $\mathbb{V}^{n}(\mu)$ restricted to the interval $[u, v]$ is constant; i.e. $\left.\mathbb{V}^{n}(\mu)\right|_{[u, v]}=\left.\mu\right|_{[u, v]}$. If $\mu([u, 1])=1$ and $\mu((v, 1])>0$ let $\mu_{1}=\left.\mu\right|_{[u, v]}, \mu_{2}=$ $\left.\mu\right|_{(v, 1]}$ and consider $A=[s(\mu)-\tau, s(\mu)]$, where $0<\tau<s(\mu)-v$. Then

$$
\begin{aligned}
\mathbb{V}(\mu)(A)= & Q\left(\mu_{1}+\mu_{2}, \mu_{1}+\mu_{2}\right)(A)=Q\left(\mu_{1}, \mu_{1}\right)(A)+2 Q\left(\mu_{1}, \mu_{2}\right)(A) \\
& +Q\left(\mu_{2}, \mu_{2}\right)(A) \\
= & 0+2 \iint P(x, y, A) d \mu_{1}(x) d \mu_{2}(y)+\iint P(x, y, A) d \mu_{2}(x) d \mu_{2}(y) \\
= & \mu([u, v]) \mu(A)+2 \int_{(v, s(\mu)-\tau)} \int_{A} P(x, y, A) d \mu_{2}(x) d \mu_{2}(y) \\
& +\int_{A} \int_{A} P(x, y, A) d \mu_{2}(x) d \mu_{2}(y) \\
\geq & \mu([u, v]) \mu(A)+\mu^{2}(A)+2\left(\frac{1}{2}+\varrho\right) \mu((v, s(\mu)-\tau)) \mu(A) \\
= & 2\left(\frac{1}{2}+\varrho\right)(1-\mu([u, v])-\mu((s(\mu)-\tau), s([\mu]))) \mu(A)+\mu^{2}(A) \\
& +\mu([u, v]) \mu(A) .
\end{aligned}
$$

Now denote $\gamma_{n}=\mathbb{V}^{n}(\mu)([s(\mu)-\tau, s(\mu)]), n=0,1, \ldots$ and $C=\mu([u, v])$. Since $\mathbb{V}^{n}([u, v])=\mu([u, v])=C$ for every natural $n$, thus by the above estimation (replace there $\mu$ by $\left.\mathbb{V}^{n}(\mu)\right)$ we get a recursion

$$
1-C \geq \gamma_{n+1} \geq 2\left(\frac{1}{2}+\varrho\right)\left(1-C-\gamma_{n}\right) \gamma_{n}+\gamma_{n}^{2}+C \gamma_{n}
$$

Now consider an elementary quadratic function $\varphi(x)=2\left(\frac{1}{2}+\varrho\right)(1-C-x) x+x^{2}+C x$ and find that for all $0<x<1-C$ we have $0<x<\varphi(x)<1-C$. Hence the iterates $\varphi^{\text {on }}(x)$ increase to $1-C$, i.e. $\lim _{n \rightarrow \infty} \mathbb{V}^{n}([s(\mu)-\tau, s(\mu)])=1-\mu([u, v])=1-C$ for all small enough $\tau>0$. It follows that for every probability measure $\mu$, concentrated on $[u, 1]$, we have 


$$
\lim _{n \rightarrow \infty} \mathbb{V}^{n}(\mu)=\left.\mu\right|_{[u, v]}+(1-\mu([u, v])) \delta_{s(\mu)}
$$

in the $\|\cdot\|_{\mathrm{FM}}$ norm.

Problem Describe weakly regular Lebesgue quadratic operators in the onedimensional case, for general symmetric (continuous) $\alpha(x, y)$, and identify limit measures.

Open Access This article is distributed under the terms of the Creative Commons Attribution 4.0 International License (http://creativecommons.org/licenses/by/4.0/), which permits unrestricted use, distribution, and reproduction in any medium, provided you give appropriate credit to the original author(s) and the source, provide a link to the Creative Commons license, and indicate if changes were made.

\section{References}

1. Alfsen, E.M.: Compact Convex Sets and Boundary Integrals. Springer, Berlin (1971)

2. Aliprantis, C.D., Burkinshow, O.: Positive Operators. Springer, Berlin (2006)

3. Barański, K., Misiurewicz, M.: Omega-limit sets for the Stein-Ulam spiral map. Topol. Proc. 36, 145-172 (2010)

4. Bartoszek, K., Pułka, M.: Asymptotic properties of quadratic stochastic operators on $L^{1}$ spaces. Nonlinear Anal. Theory Methods Appl. 114, 26-39 (2015)

5. Bartoszek, K., Pułka, M.: Prevalence problem in the set of quadratic stochastic operators acting on $L^{1}$. Bull. Malay. Math. Sci. Soc. (2015). doi:10.1007/s40840-015-0245-7

6. Bartoszek, K., Domsta, J., Pułka, M.: Centred Quadratic Stochastic Operators. arXiv:1511.07506v1

7. Bartoszek, W., Pułka, M.: On mixing in the class of quadratic stochastic operators. Nonlinear Anal. Theory Methods Appl. 86, 95-113 (2013)

8. Bernstein, S.N.: Mathematical problems in modern biology. Sci. Ukr. 1, 14-19 (1922). (in Russian)

9. Bernstein, S.N.: Solution of a mathematical problem related to the theory of inheritance. Uch. Zap. n.-i. kaf. Ukrainy 1, 83-115 (1924). (in Russian)

10. Billingsley, P.: Convergence of Probability Measures. Wiley, New York (1968)

11. Ganikhodjaev, N.: On stochastic processes generated by quadratic operators. J. Theor. Prob. 4, 639-653 (1991)

12. Ganikhodjaev, N., Ganikhodjaev, R., Jamilov, U.: Quadratic stochastic operators and zero-sum game dynamics. Ergod. Theory Dyn. Syst. 35(5), 1443-1473 (2015)

13. Ganikhodjaev, N., Mukhamedov, F.: Quantum Quadratic Operators and Processes, Lectures Notes in Mathematics, vol. 2133. Springer, Berlin (2015)

14. Ganikhodjaev, N., Saburov, M., Nawi, A. M.: Mutation and chaos in nonlinear models of heredity. Sci. World J. 2014, 1-11 (2014), arXiv:1304.5710v1

15. Ganikhodjaev, N., Saburov, M., Muhitdinov, R.: On Lebesgue nonlinear transformations. Bull. Korean Math. Soc. 54(2), 607-618 (2017)

16. Ganikhodjaev, R.: A family of quadratic stochastic operators that act in $S^{2}$. Dokl. Akad. Nauk Uz. 1, 3-5 (1989)

17. Ganikhodjaev, R., Mukhamedov, F., Rozikov, U.: Quadratic stochastic operators and processes: results and open problems. Infin. Dimens. Anal. Quantum Probab. Relat. Top. 14(2), 270-335 (2011)

18. Ganikhodjaev, R., Mukhamedov, F., Saburov, M.: Elliptic Quadratic Operator Equations. (2017). arXiv: $1701.01990 \mathrm{v} 1$

19. Jamilov, U.U., Scheutzow, M., Wilke-Berenguer, M.: On the random dynamics of Volterra quadratic operators. Ergod. Theory Dyn. Syst. 37(1), 228-243 (2017)

20. Kesten, H.: Quadratic transformations: a model for population growth I. Adv. Appl. Probab. 2, 1-82 (1970)

21. Kesten, H.: Quadratic transformations: a model for population growth II. Adv. Appl. Probab. 2, 179-228 (1970)

22. Lasota, A.: Układy dynamiczne na miarach. Wydawnictwo Uniwersytetu Ślaskiego (2008)

23. Lyubich, Y.I.: Mathematical Structures in Population Genetics. Springer, Berlin (1992) 
24. Mukhamedov, F.: Uniform stability and weak ergodicity of non homogeneous Markov chains defined on ordered Banach spaces with a base. Positivity 20(1), 135-153 (2016)

25. Mukhamedov, F., Embong, A.F.: Uniqueness of Fixed Points of b-Bistochastic Quadratic Stochastic Operators and Associated Nonhomogeneous Markov Chains. (2016). arXiv:1601.04297v1

26. Mukhamedov, F., Taha, M.H.B.M.: On Volterra and orthogonality preserving quadratic stochastic operators. Miskolc. Math. Notes 17(1), 457-470 (2016)

27. Schaefer, H.H.: Banach Lattices and Positive Operators. Springer, Berlin (1974)

28. Ulam, S.: A Collection of Mathematical Problems. Interscience, New York (1960)

29. Zakharevich, M.: On behavior of trajectories and the ergodic hypothesis for quadratic transformations of the simplex. Russ. Math. Surv. 33(6), 265-266 (1978) 